\title{
A randomized assessment of an advanced tissue preservation technology in the juvenile sheep model
}

\author{
Willem Flameng, MD, PhD, Hadewich Hermans, MD, Erik Verbeken, MD, PhD, and Bart Meuris, MD, PhD
}

\begin{abstract}
Background: Despite improved anticalcification technology, bioprosthetic heart valves still cannot be used in younger patients because of progressive structural valve degeneration. A novel advanced tissue preservation technology was developed that uses stable functional group capping and preservation by glycerolization. Valves incorporating this novel technology can be stored in a dry condition and do not require rinsing before use. The aim of the study was to assess the effects of this new technology in terms of valve function and durability in a chronic sheep model of orthotopic implantation.
\end{abstract}

Methods: Forty-five juvenile sheep were randomized and either a Perimount mitral valve (6900P, control group) or the same valve design incorporating the novel tissue preservation technology (test group) was implanted in the mitral position. All valves were $25 \mathrm{~mm}$. A transthoracic echocardiography was performed at 1 week and at 8 months postoperatively. The animals were then killed, an autopsy was performed, and the valves were examined radiographically (soft tissue radiograph), histologically (hematoxylin and eosin and Von Kossa staining), and chemically (calcium content). Exclusion criteria for analysis included surgical or procedural death, bacterial endocarditis or other diseases leading to premature death.

Results: Thirty-one animals (14 controls and 17 test animals) remained in perfect condition during the 8-month follow-up period. Echocardiography at 1 week showed normal valve function in both groups. At 8 months, cardiac output increased significantly to the same extent in both groups (vs baseline; $P<.01$ ). The mean transvalvular pressure gradient also increased but significantly more in the control group compared with the test group $(P=.03)$. Flow turbulence across the prosthesis was increased in the control valves compared with the test valves. The test valves had significantly less calcium content than the controls $(1.9 \pm 0.3 \mathrm{vs}$ $6.8 \pm 1.6 \mu \mathrm{g} / \mathrm{mg} ; P=.002$ ). This was confirmed by radiographic analysis and histology.

Conclusions: This study demonstrates that the novel tissue preservation technology, when applied to the Perimount mitral valve, significantly improves hemodynamic and anticalcification properties compared with the standard Perimount, a valve currently considered the standard of care. (J Thorac Cardiovasc Surg $2015 ; 149: 340-5)$

See related commentary on pages 346-7.

Since the Starr-Edwards heart valve was first implanted in 1960, many generations of both mechanical and bioprosthetic valves have emerged. The advantages of bioprosthetic valves include a much lower frequency of

From the Laboratory of Experimental Cardiac Surgery, Department of Cardiovascular Diseases, Katholieke Universiteit Leuven, Leuven, Belgium.

Disclosures: For this sheep study, all costs related to animal care, surgery, postoperative follow-up, and postexplant analyses, were sponsored by Edwards LifeSciences, Irvine, Calif. Apart from this, the authors have no financial relationship with Edwards LifeSciences. More specifically, the authors have no relation to the further commercial development of this new tissue preservation technology. B. Meuris has received lecture fees from the Sorin Group.

Received for publication July 13, 2014; revisions received Sept 11, 2014; accepted for publication Sept 21, 2014; available ahead of print Oct 25, 2014.

Address for reprints: Bart Meuris, MD, PhD, University Hospitals Leuven, Cardiac Surgery, Herestraat 49, B-3000 Leuven, Belgium (E-mail: bart.meuris@ uzleuven.be).

$0022-5223 / \$ 36.00$

Copyright (c) 2015 by The American Association for Thoracic Surgery

http://dx.doi.org/10.1016/j.jtcvs.2014.09.062 thromboembolism and therefore long-term anticoagulation therapy can be avoided. ${ }^{1}$ However, structural valve degeneration including calcification remains a major drawback with this type of prosthesis. Clinical data suggest that bovine pericardial valves calcify less than porcine valves. $^{2}$ This, however, does not imply that pericardial bioprostheses do not calcify. It has been extensively shown in sheep, as well as in humans, that pericardial prostheses of varying designs and from different manufacturers tend to calcify at different rates. ${ }^{3-5}$ Strong predictors of valve calcification include the stress distribution within the valve and the absence of an anticalcification treatment, which can differ considerably among valve types.,7 In general, the biological tissue used in the construction of bioprosthetic heart valve substitutes is fixed in glutaraldehyde to cross-link the tissue, thereby preventing early degeneration of the material after implantation. However, glutaraldehyde fixation is also associated with in vivo calcification of tissue. To overcome these detrimental effects, various pre- and postglutaraldehyde fixation tissue treatments have been developed. ${ }^{8,9}$ These 


\section{Abbreviations and Acronyms \\ $\mathrm{CEP}=$ Carpentier-Edwards Perimount \\ $\mathrm{EO}=$ ethylene oxide}

include detergents or surfactants, such as polysorbate 80 (Tween-80). Polysorbate 80 is the major component of the anticalcification technology used in the construction of the Carpentier-Edwards Perimount valve, which, in addition to its design characteristics that optimizes stress distribution and a special pericardial tissue assembling technique, may be responsible for its excellent long-term durability in elderly patients. ${ }^{4}$ To be considered feasible in younger patients, however, the anticalcification properties of the leaflet tissue must be enhanced further. Therefore, an innovative advanced tissue preservation technology was developed.

The aim of this study was to compare the anticalcification efficacy of this novel technology with the standard technology in juvenile sheep, which currently represents the best preclinical model to gauge the potential for bioprosthetic heart valve calcification in humans.

\section{MATERIALS AND METHODS \\ Animals}

All animals were cared for by a veterinarian in accordance with the Guide for the Care and Use of Laboratory Animals published by the National Institutes of Health (NIH publication 85-23, revised 1985). The Ethics Committee of the Katholieke Universiteit Leuven approved the study.

Forty-five female juvenile sheep, all less than 6 months of age and weighing between 22 and $38 \mathrm{~kg}$, were obtained from the Zoötechnical Center, Katholieke Universiteit Leuven, and were quarantined at the animalium facility of the Medanex Clinic (Webbekom, Belgium) before undergoing surgery. After surgery, they were kept at this facility for 8 months.

\section{Description of the Device}

The Carpentier-Edwards Perimount (CEP) mitral valve (6900P) was used in the control group and the Perimount mitral valve (6900P) incorporating the novel tissue preservation technology was used in the test group (Figure 1). All valves used were $25 \mathrm{~mm}$ in size. The leaflets of the CEP 6900P mitral valve are composed of bovine pericardium. During manufacture, the pericardial tissue thickness is measured and leaflet deflection testing is conducted to characterize each leaflet with regard to elasticity. All 3 leaflets are matched for similar thickness and elasticity, after which each leaflet is mounted onto a wireform frame to minimize commissural stress points. Bovine pericardium was selected because of its superior intrinsic properties for valve manufacture, notably in terms of collagen content and tolerance to high bending curvatures. ${ }^{2}$ The novel tissue preservation technology introduces several new approaches to preservation of tissue integrity: functional group capping, glycerolization, and a new terminal ethylene oxide (EO) sterilization process. Capping is used to reduce functional groups (eg, aldehydes) present in the glutaraldehyde-fixed tissue to prevent oxidation of the tissue and to mitigate tissue calcification. There are several ethanol rinse steps between capping and glycerolization that lower the amount of residual chemicals to acceptable levels. The other novel aspect of the technology is that the valves undergo treatment with a glycerol and ethanol mix, which displaces most of the water present in the pericardial tissue and replaces it with glycerol. As a result of glycerolization, the valves can be packaged and stored dry, without the need for any liquid-based storage solution such as glutaraldehyde. The valves are sterilized via 100\% EO. Rinsing is not required before use.

\section{Surgery}

The animals were operated under general anesthesia. The animals were premedicated with ketamine $(10-20 \mathrm{mg} / \mathrm{kg}$ body weight, intramuscularly). Anesthesia was induced with increasing concentrations of isoflurane (Isoba, Schering-Plough Animal Health, Middlesex, United Kingdom) in oxygen. Anesthesia was maintained with halothane and $\mathrm{N}_{2} \mathrm{O}$. After endotracheal intubation, mechanical ventilation was started. All ventilation parameters were adjusted to keep the arterial blood gasses and $\mathrm{pH}$ within the physiologic range. A large-bore orogastric tube was placed in the rumen, and allowed to drain by gravity to prevent rumenal distention. Electrocardiographic limb leads were connected and monitored. A maintenance intravenous drip of Ringer solution was started. Gentamycin (6.6 mg/kg, Genta-Kel 10\%, Hoogstraten, Belgium) and benzylpenicillinum natricum $(40,000 \mathrm{U} / \mathrm{kg}$, penicillin, 1,000,000 units, Kela, Hoogstraten, Belgium) was administered venously. A left thoracotomy was carried out, then the animals were placed on cardiopulmonary bypass and mitral valve replacement was performed as described previously. ${ }^{3}$ The animal was placed on the operating table in the right lateral recumbent position. The sheep was surgically scrubbed and draped to expose the distal left cervical region. An arterial pressure monitoring line was placed in the right central ear artery. A baseline blood sample was drawn. The left carotid artery and the jugular vein were freed from surrounding tissue. Afterwards, the left chest was prepped and draped, and a left thoracotomy was performed in the third interspace. The ribs were retracted for adequate exposure of the heart and the pericardium was incised in a T-shaped fashion anterior to the phrenic nerve. The heart was suspended in a pericardial cradle by suspension of the pericardial flaps. After administration of heparin $(3 \mathrm{mg} / \mathrm{kg})$, the carotid artery and the jugular vein were cannulated with an arterial cannula and a 2stage venous cannula of appropriate size and connected to the heartlung machine. Extracorporeal circulation was started and maintained at an adequate flow rate without cooling. Then, the heart was electrically fibrillated and the auricle of the left atrium was incised. The mitral valve was exposed and the left ventricle was emptied by suction through the mitral valve. The valve was implanted similar to a clinical situation (with separate, pledgeted, 2/0 Premicron sutures). After implantation of the valve, the left atrium was closed and the heart defibrillated. When the hemodynamics were stable, the heart-lung machine was stopped. The cannulas were removed and the vessels ligated. The wound in the neck was closed in layers and careful hemostasis was performed. The chest was closed in layers with a chest drain in the left pleural space. The valve was chosen immediately before surgery by picking an envelope by the study assistant.

\section{Postoperative Care}

The animal was weaned from the ventilator as soon as there was spontaneous respiration with adequate tidal volumes and stable hemodynamics. The animal was carefully observed during the immediate postoperative period for up to 7 days. The chest drain was removed after 3 hours when possible. The animals receive analgesics (piritramide) for the first 3 days on regular schemes as well as diuretics. Albipen LA and enoxaparine (20 mg twice daily) were administered for 3 days. Afterwards, the animals returned to the controlled animal facility where the general health of the sheep was checked daily. 

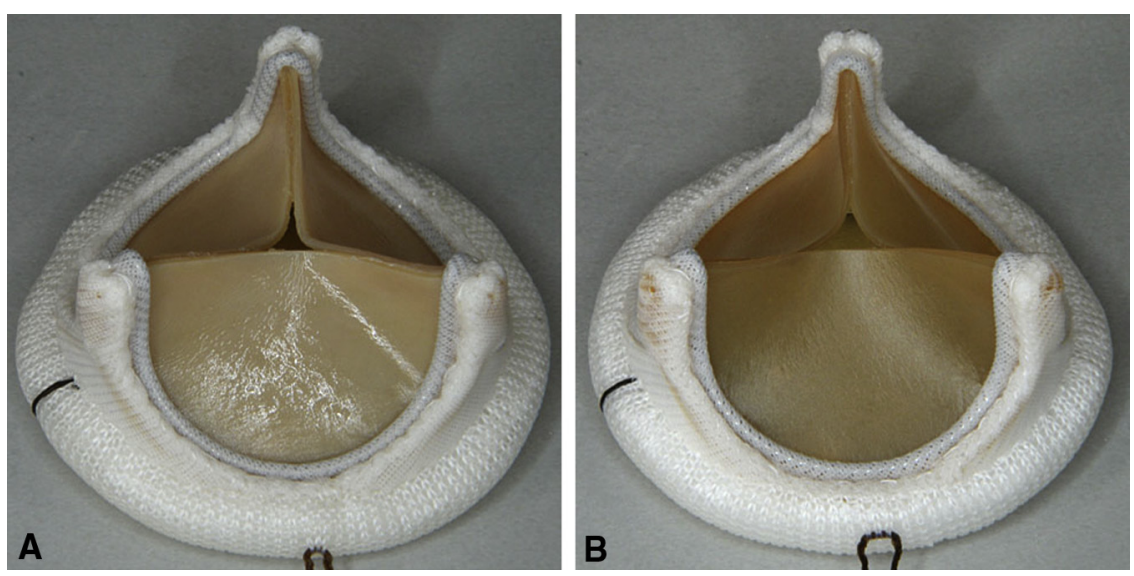

FIGURE 1. Study devices. CEP mitral bioprosthesis 6900P (A) and the CEP mitral bioprosthesis with the novel technology (B). The valve in B is stored under dry conditions in a sterile container.

\section{Echocardiography}

At 1 week (postoperative control) and at 8 months (before explantation of the valve) postoperatively, a transthoracic echocardiography was performed under mild sedation. ${ }^{10}$ An experienced clinical cardiologist, blinded to the identity of the group, performed all echocardiographic examinations. The following measurements/observations were performed: mean and peak transvalvular gradient, regurgitation grade, leaflet mobility, and flow turbulence (graded as laminar, mild, moderate, or severe).

\section{Early Deaths}

Any animals that died before the 8-month explant period underwent full necropsy within 12 to 24 hours post mortem. A complete necropsy was performed and an attempt to determine the cause of death was made.

\section{Explant Procedures}

The animals were killed at 8 months. The animal was prepared and anesthetized as described earlier. After the echocardiographic assessment, the sheep received heparin $(3 \mathrm{mg} / \mathrm{kg})$ intravenously and was killed with an overdose of a euthasol solution intravenously and the heart was removed. The implanted bioprosthesis was excised and analyzed as described in the following section.

\section{Examination of the Valve}

Gross examination. After careful rinsing the explanted tissue, the following macroscopic parameters were evaluated and scored: vegetation, cuspal hematoma, thrombosis, tears or perforations, tissue overgrowth (fibrous sheating), paravalvular leak, visible calcification, and cusp retraction. Macroscopic photographs of the valves were taken.

Radiographic assessment. Radiographic examinations were performed under mammography conditions (soft tissue radiograph; Faxitron, Tucson, Ariz) to demonstrate and localize calcifications. The severity of calcification was scored as absent or present.

Dissection of the explanted valve. The leaflets were separated from the mounting frame and identified according to their implant localization: anterior, posterior directed to the posterior papillary muscle, and posterior directed to the anterior papillary muscle. From the middle part of each leaflet, a longitudinal section was taken from the free edge to the base and sent for histologic examination. The rest of the leaflet was assessed for calcium content.

Histologic data. For histology, 5- $\mu$ m-thick cross-sections were prepared from the leaflet. The sections were embedded in paraffin. All sections were routinely stained with hematoxylin and eosin and Von Kossa calcium staining. When necessary, additional sections and/or stainings were made. Using light microscopy, the histologic integrity of the tissue, the localization and the extent of calcification, the presence of an inflammatory response in the tissue, and the extent of fibrous sheeting or pannus over the valve tissue were evaluated.

Quantitative calcium determination. Calcium content was quantified spectrophotometrically using a calcium colorimetric Kit (Chema Diagnostica, Monsano, Italy) and expressed as micrograms per milligram dry weight.

\section{Data Management and Statistical Analysis}

Values are represented as means \pm standard deviation, or as proportions, as appropriate. Means were compared using a standard $t$ test, proportions were compared using the Fisher exact test.

\section{RESULTS}

Forty-five juvenile sheep were allocated at random to a control group (group $\mathrm{C}, \mathrm{n}=23$ ), which received a commercially available standard size 25 Perimount mitral valve (6900P), and a test group (group $\mathrm{T}, \mathrm{n}=22$ ), which received a size 25 Perimount mitral valve (6900P) with the novel tissue preservation technology.

\section{Mortality and Morbidity}

Eight animals died as a result of the surgical intervention or anesthesia: 4 animals in group $\mathrm{C}$ and 4 animals in group $\mathrm{T}$. In some of these juvenile animals (body weight between 22 and $38 \mathrm{~kg}$ ), the $25-\mathrm{mm}$ prosthesis was too large for the mitral annulus, leading to valve dehiscence, severe paravalvular leakage, and/or ventricular dissection.

Four animals $(8.8 \%)$ developed prosthetic valve endocarditis and died during follow-up. This was diagnosed by the general condition of the animal (fever, sepsis, dyspnea, a new heart murmur) and confirmed by echocardiography and/or autopsy (including histology of the valves). This occurred in 3 animals in group $C$ and in 1 in group $\mathrm{T}$ between 2 weeks and 7 months postoperatively. 
TABLE 1. Echocardiographic measurements at 1 week and 8 months of follow-up in both groups

\begin{tabular}{|c|c|c|c|c|}
\hline & \multicolumn{2}{|c|}{ Group C } & \multicolumn{2}{|c|}{ Group T } \\
\hline & 1 Week & 8 Months & 1 Week & 8 Months \\
\hline Heart rate (bpm) & $95.1 \pm 21.2$ & $101.8 \pm 24.3$ & $101.7 \pm 21.1$ & $96.0 \pm 17.7$ \\
\hline Cardiac output (L/min) & $3.6 \pm 1.3$ & $5.1 \pm 1.4^{*}$ & $3.9 \pm 0.9$ & $4.9 \pm 0.9^{*}$ \\
\hline Mean pressure gradient $(\mathrm{mm} \mathrm{Hg})$ & $2.1 \pm 0.8$ & $5.5 \pm 2.3^{*}$ & $2.1 \pm 0.9$ & $3.9 \pm 1.6^{*}$, \\
\hline Peak pressure gradient $(\mathrm{mm} \mathrm{Hg})$ & $5.1 \pm 1.5$ & $10.8 \pm 4.2^{*}$ & $4.6 \pm 1.7$ & $8.1 \pm 3.6^{*}$ \\
\hline
\end{tabular}

$* P<.01$ versus the measurements at 1 week. $\dagger P=.03$ versus the mean gradient in group $\mathrm{C} . \ddagger P=.06$ versus the peak gradient in group $\mathrm{C}$.

During follow-up, 2 more animals died ( 1 in group $\mathrm{C}$ and 1 in group T) at 7 months postoperatively. They had recurrent symptoms of fever, tachypnea, dyspnea, and eventually renal failure and a severe heart murmur. There was no clear diagnosis of endocarditis on the prosthesis but these animals had to be excluded from the analysis because they did not meet the required criteria of eventfree survival for 8 months.

Thus, 14 animals in group $\mathrm{C}$ and 17 animals in group Tall remained in perfect condition during 8 months of follow-up.

\section{Hemodynamic Evaluation of the Valves}

Control echocardiography of these 31 animals revealed perfect valve function at 1 week in all control and test animals (Table 1). All valves showed perfect leaflet coaptation and laminar flow or at the most some mild turbulence over the valve. At 8 months, cardiac output was increased in group $\mathrm{C}$ to $5.1 \mathrm{~L} / \mathrm{min}$ and in group $\mathrm{T}$ to $4.9 \mathrm{~L} / \mathrm{min}$ (Table 1 ). Mean and peak gradients over the valve increased in both groups during the 8 months of follow-up, in parallel to the increase in cardiac output, but not to the same extent in both groups. Gradients in group $\mathrm{C}$ increased significantly more than gradients in group $\mathrm{T}(P=.03$ for the mean gradient and $P=.06$ for the peak gradient) (Figure 2, A). At 8 months, 9 of 14 valves in group $\mathrm{C}$ developed moderate to severe turbulence over the valve, compared with only 1 of 17 valves in group $\mathrm{T}(P=.0008)$. In group $\mathrm{C}, 7$ of 14 valves showed malcoaptation compared with 3 of 17 in group $\mathrm{T}$ $(P=.06)$.

\section{Calcium Content of the Valves}

The calcium content in the leaflets of the valves in group C was $6.8 \pm 1.6 \mu \mathrm{g} / \mathrm{mg}$ compared with $1.9 \pm 0.3 \mu \mathrm{g} / \mathrm{mg}$ in group T $(P=.002$, Figure $2, B)$.

\section{Radiographic Examination of the Valves}

Faxitron radiographic analysis of the explanted valves revealed visible calcifications and offered insight into the spatial distribution of calcific deposits throughout the valve. In group C, only 6 of 14 valves were free from any leaflet calcification (43\%) compared with 13 of 17 valves in group $\mathrm{T}(76 \%, P=.06)$. In group $\mathrm{C}$, only 7 of 14 valves were free of commissural calcifications (50\%) compared with 11 of 17 valves in group $\mathrm{T}(65 \%, P=.3)$. The number of calcified leaflets per valve differed between the groups (Figure 3). Two representative examples of radiographic images are shown in Figure 4.

\section{Histologic Analysis of the Valve Leaflets}

Based on staining as described earlier, the presence of fibrin deposits, extrinsic and intrinsic calcification, neointima formation, and signs of inflammation could be identified on the complete sections of the middle part of each valve leaflet. Neointima formation was found in all leaflets in all valves, irrespective of the group. Neointima formation was mainly found on the atrial side of the leaflets. In group C, 4 of 14 valves also showed signs of inflammation, characterized by the presence of giant cells in the leaflet. This was never found in valves in group
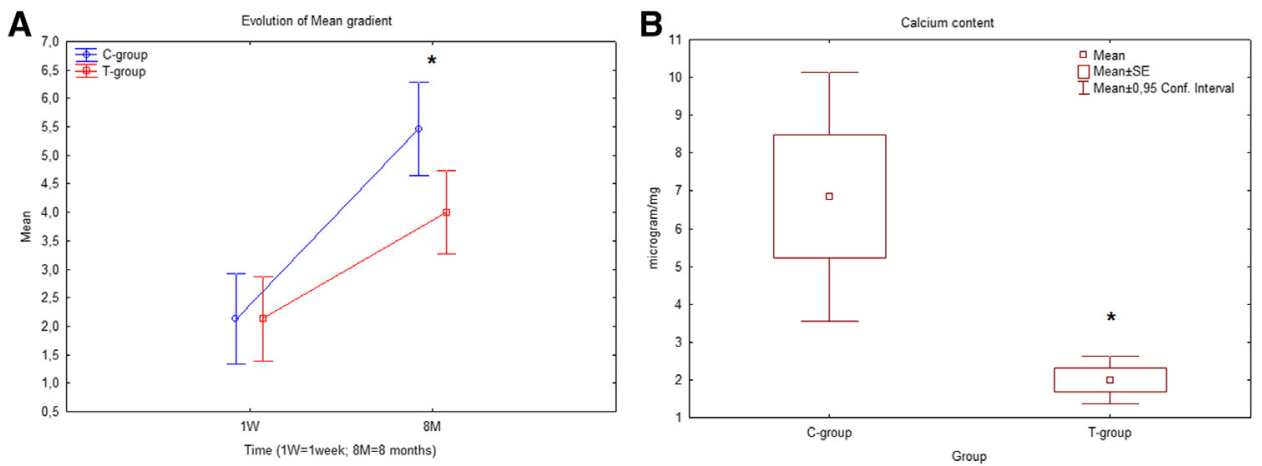

FIGURE 2. A, Evolution in mean transvalvular gradients (mean \pm standard error of the mean) from 1 week to 8 months in both groups $(* P=.03$ at 8 months). B, Final calcium content of both valve types $(* P=.002) . S E$, Standard error. 


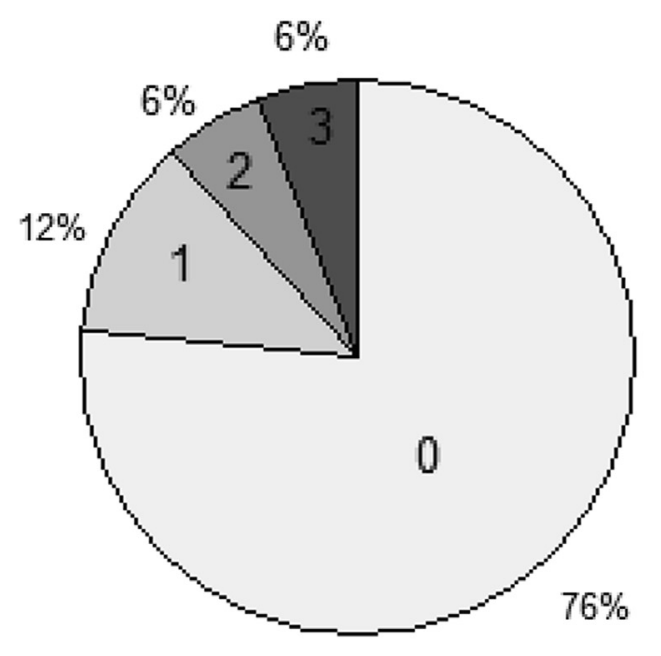

T-group: Leaflets

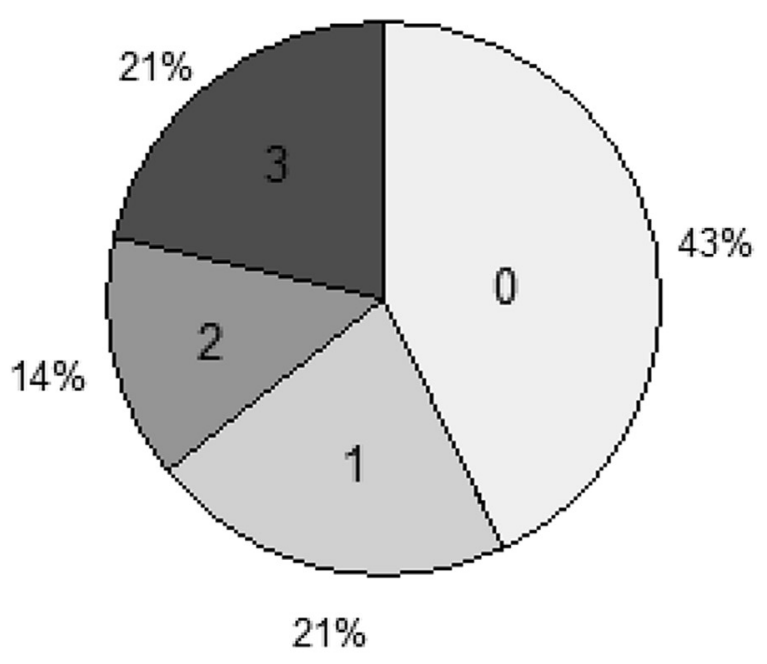

C-group: Leaflets

FIGURE 3. Radiographic scoring for all leaflets of both valve types. 0 , fraction of valves with all leaflets free from any visible mineralization; 1 , fraction of valves with 1 calcified leaflet; 2 , fraction of valves with 2 calcified leaflets; 3 , fraction of valves with 3 calcified leaflets.

T. Extrinsic calcifications were found in valves in both groups: in 2 of 14 valves in group $\mathrm{C}$ and 3 of 17 valves in group T. However, intrinsic calcifications of the leaflets were only found in group $\mathrm{C}$ and never in group $\mathrm{T}$ ( 4 of 14 vs 0 of 17).

\section{Blood Analysis}

Blood samples taken preoperatively, at 1 week postoperatively, and at 8 months were analyzed for hemoglobin, hematocrit, red blood cells, platelets, and white blood cells. In both groups, hemoglobin, hematocrit, and red blood cell count were significantly higher at 8 months postoperatively compared with preoperatively $(P<.05)$. The white blood cell count was increased in both groups at 1 week, but normalized thereafter. No significant differences were detected in the hematologic parameters between the animals in groups $\mathrm{C}$ and $\mathrm{T}$.

\section{Autopsy Results}

The general autopsy results did not reveal any abnormalities in any of the surviving animals. Specific macroscopic inspection of the implanted valves showed the absence of valve thrombosis, cuspal hematoma, vegetation, tears or perforations, or paravalvular leakage in any of the valves. All valves had some signs of tissue overgrowth, mainly on the atrial side. Cusp retraction was found in only 1 valve in group $\mathrm{C}$.

\section{DISCUSSION}

Based on the favorable long-term clinical experience with the CEP bioprosthetic heart valve, many surgeons consider it one of the standard of care valves. Outcomes from long-term observational studies have reported low mortality and morbidity rates, low risk of structural valve degeneration, and a low incidence of patient-prosthesis mismatch. All these advantages can be attributed to the valve design and anticalcification technology. ${ }^{11-13}$ Nonetheless, the use of the Perimount valve remains primarily limited to elderly patients because of an increasing incidence of structural valve degeneration in recipients younger than 60 years of age.

The bovine pericardial tissue used in the construction of the current Perimount valve is cross-linked using glutaraldehyde. The tissue is then processed through a solution of formaldehyde, ethanol, and Polysorbate- 80 Tween. Glutaraldehyde has been shown to reduce both the antigenicity of xenograft tissue and increase its stability. ${ }^{2,14}$

The novel tissue preservation technology used in the test valves introduces important innovations: functional group capping, glycerolization, and EO sterilization. It is well known that glutaraldehyde can provoke or initiate tissue calcification. Therefore, some research groups are focusing on completely avoiding the use of glutaraldehyde in the preparation of biological tissue. ${ }^{15}$ The technology used in this study does not avoid glutaraldehyde but has a permanent free aldehyde capping and reduction process in the treatment sequence. The ethanol rinses within the process are designed to remove residual chemicals as the valves go through processing. The reduction in phospholipids occurs through a separate process that uses ethanol and Tween for an extended (proprietary) soak time, to remove phospholipids and cellular debris from the tissue.

There are no other differences between the test and control valves with regard to the sewing ring, stent, tissue thickness, or tissue attachment. Both test and control valves are built the same way; the only difference is in how the valves are treated after assembly. The novel technology 

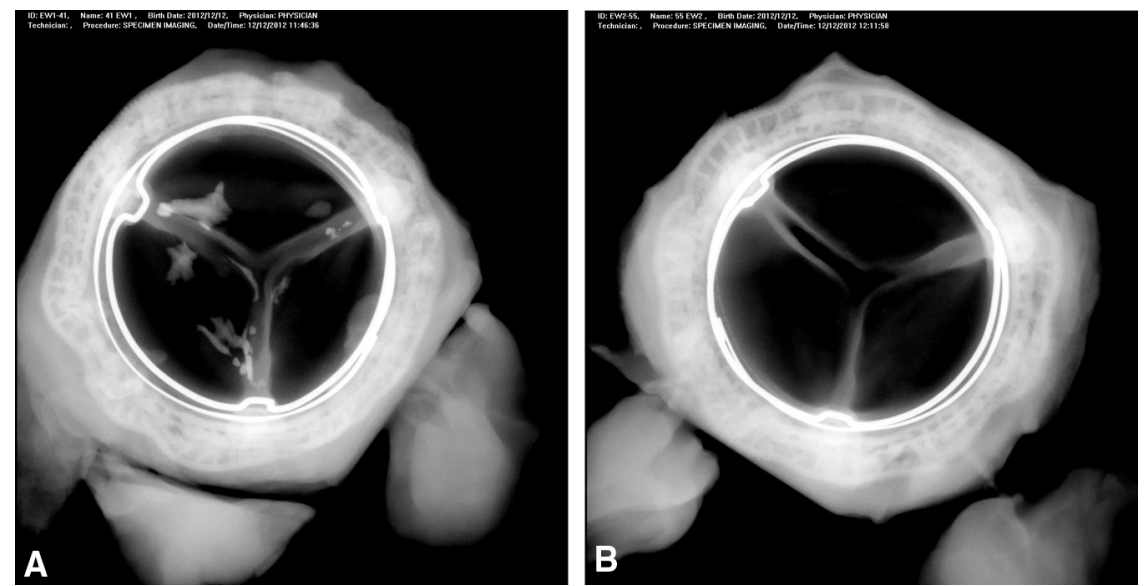

FIGURE 4. Explanted valves, Radiographic analysis from group C (A) and group $\mathrm{T}$ (B). Clear commissural and leaflet calcifications can be seen in the valve from group $\mathrm{C}$.

enables the valve to be packaged and stored dry without the need for a liquid storage solution.

A juvenile sheep model with orthotopic valve implantation in animals less than 6 months of age was used. This model mirrors the accelerated calcification that is often seen in younger humans. Using such a model, important biological tissue calcification can be observed (after only 5 months of implantation, which is the usual term required by the US Food and Drug Administration) in various bioprostheses, certainly when no or less optimal anticalcification strategies are used. ${ }^{3}$ With a mean calcium level of only $6.8 \mu \mathrm{g} / \mathrm{mg}$, the current Perimount valve performed well in this long-term 8-month study in the mitral position. But the valves in the test group did even better, reaching only $1.9 \mu \mathrm{g} / \mathrm{mg}$. Also the semiquantitative calcium scores on radiography revealed a better result for the test valves.

Transthoracic echocardiography within this long-term sheep study was performed by a cardiologist, who was blinded to the identity of the group. By studying the evolution in cardiac output and transvalvular gradient in both groups, we showed that valve function was improved in the test group. The gradient across the valve is expected to increase as the normal cardiac output increases with somatic growth, however, it usually increases even more when leaflet compliance diminishes because of progressive calcification and/or stiffening of the tissue. This phenomenon appeared to be counteracted by the novel tissue technology, with lower mean transvalvular gradients in the test group and lower flow turbulence.

In conclusion, we demonstrate that a novel tissue preservation technology, when applied to the Perimount mitral valve, significantly improved the hemodynamic and anticalcification properties compared with a standard Perimount, a valve, currently considered the standard of care.

\section{References}

1. Carpentier A, Dubost C, Lane E, Nashef A, Carpentier S, Relland J, et al Continuing improvements in valvular bioprostheses. J Thorac Cardiovasc Surg. 1982;83:27-42.

2. Liao K, Seifter E, Hoffman D, Yellin EL, Frater RW. Bovine pericardium versus porcine aortic valve: comparison of tissue biological properties as prosthetic valves. Artif Organs. 1992;16:361-5.

3. Flameng W, Meuris B, Yperman J, De VG, Herijgers P, Verbeken E. Factors influencing calcification of cardiac bioprostheses in adolescent sheep. $J$ Thorac Cardiovasc Surg. 2006;132:89-98.

4. Flameng W, Rega F, Vercalsteren M, Herijgers P, Meuris B. Antimineralization treatment and patient-prosthesis mismatch are major determinants of the onset and incidence of structural valve degeneration in bioprosthetic heart valves. J Thorac Cardiovasc Surg. 2014;147:1219-24.

5. Jones M, Eidbo EE, Hilbert SL, Ferrans VJ, Clark RE. Anticalcification treatments of bioprosthetic heart valves: in vivo studies in sheep. J Card Surg. 1989;4:69-73.

6. Carpentier A. Hemodynamic factors affecting the fate of valvular bioprosthesis Circulation. 2010;121:2083-4.

7. Flameng W, Herregods MC, Vercalsteren M, Herijgers P, Bogaerts K, Meuris B. Prosthesis-patient mismatch predicts structural valve degeneration in bioprosthetic heart valves. Circulation. 2010;121:2123-9.

8. Carpentier A, Nashef A, Carpentier S, Ahmed A, Goussef N. Techniques for prevention of calcification of valvular bioprostheses. Circulation. 1984;70(3 Pt 2):I165-8

9. Girardot MN, Torrianni M, Girardot JM. Effect of AOA on glutaraldehyde-fixed bioprosthetic heart valve cusps and walls: binding and calcification studies. Int J Artif Organs. 1994;17:76-82.

10. De Vleeschauwer S, De Praetere H, Meuris B, Herijgers P, Herregods MC Post-operative echocardiographic evaluation of bioprosthetic mitral valve implantation in sheep. Lab Anim. August 12, 2014 [Epub ahead of print].

11. Ayegnon KG, Aupart M, Bourguignon T, Mirza A, May MA, Marchand M A 25-year experience with Carpentier-Edwards Perimount in the mitral position. Asian Cardiovasc Thorac Ann. 2011;19:14-9.

12. Flameng W, Meuris B, Herijgers P, Herregods MC. Prosthesis-patient mismatch is not clinically relevant in aortic valve replacement using the Carpentier-Edwards Perimount valve. Ann Thorac Surg. 2006;82:530-6.

13. Frater RW, Furlong P, Cosgrove DM, Okies JE, Colburn LQ, Katz AS, et al. Long-term durability and patient functional status of the Carpentier-Edwards Perimount pericardial bioprosthesis in the aortic position. J Heart Valve Dis. 1998;7:48-53.

14. Vesely I, Mako WJ. Comparison of the compressive buckling of porcine aortic valve cusps and bovine pericardium. J Heart Valve Dis. 1998;7:34-9.

15. Barros JA, Filippin-Monteiro FB, de Oliveira EM, Campa A, Catalani LH, Pitombo RN, et al. Cytotoxicity of PVPAC-treated bovine pericardium: a potential replacement for glutaraldehyde in biological heart valves. J Biomed Mater Res B Appl Biomater. 2014;102:574-82. 